PROCEEDINGS OF THE

AMERICAN MATHEMATICAL SOCIETY

Volume 126, Number 3, March 1998, Pages 819-822

S $0002-9939(98) 04167-7$

\title{
A VISIT TO THE ERDŐS PROBLEM
}

\author{
PAUL D. HUMKE AND MIKLÓS LACZKOVICH
}

(Communicated by J. Marshall Ash)

\begin{abstract}
Erdős asked if for every infinite set, $A$, of real numbers there exists a measurable subset of the reals having positive measure that does not contain a subset similar to $A$. In this note we transform this question to a finite combinatorial problem. Using this translation we extend some results of Eigen and Falconer concerning slow sequences for which the answer to Erdös' question is positive.
\end{abstract}

We first establish some notation. Let $\mathbb{N}_{n}=\{1,2, \ldots, n\}$. The integer part of $y$ is denoted by $[y]$. For $A, B \subset \mathbb{R}$ the notation $A \sim B$ means that there are $u, v \in \mathbb{R}$ with $v \neq 0$ such that $A=u+v \cdot B=\{u+v b: b \in B\}$ and in this case we say that $A$ is similar to $B$. If $A \sim B$ with $v>0$, then $A$ is said to be homothetic to $B$ and this is denoted by $A \approx B$.

Let $A \subset[0,1]$ be given. Define $\Lambda_{n}$ as the cardinality of the smallest set $B \subset \mathbb{N}_{n}$ that intersects each subset of $\mathbb{N}_{n}$ of the form

$$
A_{x, y}=\{x+[a y]: a \in A\} \text {, where } x, y, x+y \in \mathbb{N}_{n} \text { and } y \geq[n / 2] .
$$

Theorem 1. Let $A \subset[0,1]$ be such that $\inf (A)=0$ and $\sup (A)=1$. Then the following are equivalent.

1. There is a measurable set of positive measure which contains no subset similar to $A$.

2. $\lim _{n \rightarrow \infty} \frac{\Lambda_{n}}{n}=0$.

3. $\liminf _{n \rightarrow \infty} \frac{\Lambda_{n}}{n}=0$.

Proof. We first prove that 1 implies 2 . Let $\varepsilon>0$ be fixed and suppose there is a set of positive measure which contains no subset similar to $A$. Then there is a closed set, $F=F(\varepsilon) \subset[0,1]$ such that $\lambda(F)>1-\varepsilon$ and $F$ contains no subset which is similar to $A$. Set

$$
B_{n}=\left\{i \in \mathbb{N}_{n}:\left[\frac{i-1}{n}, \frac{i}{n}\right] \cap F=\emptyset\right\} .
$$

Then $\left|B_{n}\right|<\varepsilon \cdot n$ for all $n$. We show that for $n$ large enough, $B_{n} \cap A_{x, y} \neq \emptyset$ whenever $x, y, x+y \in \mathbb{N}_{n}$ and $y \in[n / 2, n]$. This will prove that $\Lambda_{n} \leq\left|B_{n}\right|<\varepsilon n$ for $n$ large enough; that is, 2 holds.

Received by the editors March 6, 1996 and, in revised form, September 9, 1996.

1991 Mathematics Subject Classification. Primary 28A99; Secondary 28A05.

The first author was supported by the National Research Council of the United States, and the second author by the Hungarian National Foundation for Scientific Research, Grant T016094.

(C)1998 American Mathematical Society 
If this is not the case, then there is a sequence $\left\{\left(n_{k}, x_{k}, y_{k}\right)\right\}$ such that for every $k \in \mathbb{N}, B_{n_{k}} \cap A_{x_{k}, y_{k}}=\emptyset$, and $x_{k}, y_{k}, x_{k}+y_{k} \in \mathbb{N}_{n_{k}}$ and $\left[\frac{n_{k}}{2}\right] \leq y_{k}$. Selecting a subsequence, we may assume $\left(x_{k} / n_{k}\right) \rightarrow x_{0}$ and $\left(y_{k} / n_{k}\right) \rightarrow y_{0} \geq \frac{1}{2}$.

Now, let $a \in A$. As $x_{k}+\left[a y_{k}\right] \notin B_{n_{k}}, F \cap I_{k} \neq \emptyset$, where

$$
I_{k}=\left[\frac{x_{k}+\left[a y_{k}\right]-1}{n_{k}}, \frac{x_{k}+\left[a y_{k}\right]}{n_{k}}\right] \text {. }
$$

But, $\left(x_{k} / n_{k}\right)+\left(a y_{k} / n_{k}\right)-\left(1 / n_{k}\right) \in I_{k}$ for each $k \in \mathbb{N}$ and hence, $x_{0}+a y_{0} \in F$ since $F$ is closed. As this is true for every $a \in A$, it follows that $x_{0}+A \cdot y_{0} \subset F$ which contradicts the choice of $F$. This completes the first part of the proof of the theorem. As the proof that 2 implies 3 is obvious, we turn to the proof that 3 implies 1 .

Fix $A$ and suppose that $\liminf _{n \rightarrow \infty} \frac{\Lambda_{n}}{n}=0$. It is enough to show that there is a measurable set, $F \subset[0,1]$, of measure greater than $1 / 2$ containing no subset which is homothetic to $A$, since in this case $F \cap(1-F)$ is a measurable set of positive measure which contains no subset similar to $A$.

Let $\varepsilon>0$ be given. We first construct an open set $G_{\varepsilon}$ of measure less than $\varepsilon$ such that if $A \approx A^{\prime} \subset[0,1]$ and $\operatorname{diam} A^{\prime} \geq \frac{1}{2}$, then $A^{\prime} \cap G_{\varepsilon} \neq \emptyset$.

As $\liminf \operatorname{in}_{n \rightarrow \infty} \frac{\Lambda_{n}}{n}=0$, for each $k=1,2, \ldots$ there are an $n_{k}$ and $B_{n_{k}} \subset \mathbb{N}_{n_{k}}$ with the required property such that $\left|B_{n_{k}}\right|<\varepsilon n_{k} / 8 \cdot 2^{k}$. Let

$$
G_{\varepsilon}=\bigcup_{k=1}^{\infty} \bigcup_{m \in B_{n_{k}}}\left(\frac{m-4}{n_{k}}, \frac{m+4}{n_{k}}\right) .
$$

Then $\lambda\left(G_{\varepsilon}\right)<\sum_{k=1}^{\infty} \frac{\varepsilon}{2^{k}}=\varepsilon$. Suppose $A^{\prime} \subset[0,1], A^{\prime} \approx A$, $\operatorname{diam} A^{\prime} \geq 1 / 2$. Then, $A^{\prime}=u+v \cdot A$ where $u, v \in[0,1]$ with $v \geq 1 / 2$ and as $\sup A=1, u+v \leq 1$. We show that $A^{\prime} \cap G_{\varepsilon} \neq \emptyset$. Put $x_{k}=\max \left(1,\left[n_{k} u\right]\right)$ and $y_{k}=\max \left(\left[n_{k} / 2\right],\left[n_{k} v\right]-1\right)$. Then $x_{k}, y_{k}, x_{k}+y_{k} \in \mathbb{N}_{n_{k}}$, and $y_{k} \geq\left[n_{k} / 2\right]$. Thus, by the choice of $B_{n_{k}}$, we have

$$
A_{x_{k}, y_{k}} \cap B_{n_{k}} \neq \emptyset \text {. }
$$

If $m \in A_{x_{k}, y_{k}} \cap B_{n_{k}}$, then there is an $a \in A$ such that $m=x_{k}+\left[a y_{k}\right]$, and it follows that

$$
\begin{aligned}
n_{k} u+a n_{k} v-4 & \leq\left(n_{k} u-1\right)+a\left(n_{k} v-2\right)-1<m \\
& \leq\left(n_{k} u+1\right)+a n_{k} v<n_{k} u+a n_{k} v+4 .
\end{aligned}
$$

Hence, $(m-4) / n_{k}<u+a v<(m+4) / n_{k}$. However, if $m \in B_{n_{k}}$ then $\left((m-4) / n_{k},(m+4) / n_{k}\right) \subset G_{\varepsilon}$ and thus $a+u v \in A^{\prime} \cap G_{\varepsilon}$. This completes the construction of $G_{\varepsilon}$.

Fix a number $c \in(1 / 2,1)$, and let $\delta_{n}=2 c^{n}-c^{n-1}$. For each $n=1,2, \ldots$ define

$$
G_{\varepsilon}^{n}=[0,1] \cap \bigcup_{k=0}^{\infty}\left(\left(2 c^{n} \cdot G_{\varepsilon}\right)+k \delta_{n}\right) .
$$

Then $G_{\varepsilon}^{n}$ intersects every set $A^{\prime} \subset[0,1]$ with $A^{\prime} \approx A$ and $c^{n} \leq \operatorname{diam} A^{\prime} \leq c^{n-1}$. Indeed, if $u=\inf A^{\prime}, v=\sup A^{\prime}$ and $k$ is the largest integer such that $k \delta_{n} \leq u$, then $[u, v] \subset 2 c^{n} \cdot[0,1]+k \delta_{n}$ since

$$
v=(v-u)+u \leq c^{n-1}+(k+1) \cdot \delta_{n}=2 c^{n}+k \delta_{n} .
$$

Let $A^{\prime \prime}=\left(A^{\prime}-k \delta_{n}\right) / 2 c^{n}$. Then $A^{\prime \prime} \subset[0,1]$ and diam $A^{\prime \prime} \geq 1 / 2$; therefore $A^{\prime \prime} \cap G_{\varepsilon} \neq$ $\emptyset$. Then it follows that $A^{\prime}$ intersects $2 c^{n} \cdot G_{\varepsilon}+k \delta_{n}$ and hence, $A^{\prime}$ intersects $G_{\varepsilon}^{n}$. 
Choose $\varepsilon_{n}>0$ such that $\lambda\left(G_{\varepsilon_{n}}^{n}\right)<2^{-n-1}$ and set

$$
G=\bigcup_{n=1}^{\infty} G_{\varepsilon_{n}}^{n}
$$

Then, $\lambda(G)<1 / 2$ and if $A \approx A^{\prime} \subset[0,1]$ then $A^{\prime} \cap G \neq \emptyset$. The proof is completed by letting $F$ be $[0,1] \backslash G$.

For $A \subset \mathbb{R}$ and $u<v$ we shall denote by $A(u, v)$ the length of the longest component of $(u, v) \backslash A$.

Theorem 2. Let $A$ be a bounded set such that

$$
\inf _{u<v} \frac{A(u, v)}{v-u}=0 .
$$

Then there is a measurable set of positive measure which contains no subset similar to $A$.

Proof. We may assume $\inf A=0$ and $\sup A=1$. Let $0<\varepsilon<1$ be given. There exist $u, v \in A$ such that $u<v$ and $|I|<\varepsilon(v-u)$ whenever $I \subset(u, v) \cap A^{c}$. Let $n=[6 / \varepsilon(v-u)]$ and $B=\{k b: k=1,2, \ldots\} \cap \mathbb{N}_{n}$ where $b=[1 / \varepsilon]$. We claim that each set $A_{x, y}$ contains a sequence of at least $b$ consecutive integers and hence, intersects $B$. Indeed, $A_{x, y} \supset\{x+[a y]: a \in[u, v] \cap A\}=A^{*}$, and using the fact that $y \geq n / 2$,

$$
\operatorname{diam} A^{*}=[v y]-[u y] \geq v y-u y-1 \geq(v-u) \frac{n}{3}>b .
$$

Since each component of $(u, v) \backslash A$ is shorter than $\frac{\varepsilon}{4}(v-u)<1 / n \leq 1 / y$ it is clear that $A^{*}$ and hence $A_{x, y}$ contains every integer between $x+[u y]$ and $x+[v y]$. Hence, $\liminf _{n \rightarrow \infty} \frac{\Lambda_{n}}{n}<\varepsilon$ and as $\varepsilon>0$ is arbitrary, $\liminf _{n \rightarrow \infty} \frac{\Lambda_{n}}{n}=0$ and Theorem 1 can be applied.

The following result was proved independently by Eigen [2] and Falconer [7], and is an immediate corollary of Theorem 2 .

Corollary 1 (Eigen [2] and Falconer [7]). Suppose $\left\{a_{n}\right\}$ is a decreasing sequence of positive numbers converging to 0 with

$$
\lim _{n \rightarrow \infty} \frac{a_{n+1}}{a_{n}}=1 .
$$

Then there is a measurable set of positive measure which contains no subset similar to $\left\{a_{n}\right\}$.

Our next theorem extends this result.

Corollary 2. Suppose $\left\{a_{n}\right\}$ is a decreasing sequence of positive numbers converging to 0 with $\left\{a_{n}-a_{n+1}\right\}$ monotonic, and $\lim _{\sup _{n \rightarrow \infty}} \frac{a_{n+1}}{a_{n}}=1$. Then there is a measurable set of positive measure which contains no subset similar to $\left\{a_{n}\right\}$.

Proof. If $\frac{a_{n+1}}{a_{n}}>1-\varepsilon$ then the length of any component of $\left[0, a_{n}\right] \backslash\left\{a_{i}\right\}$ is less than $\varepsilon a_{n}$ and so Theorem 2 can be applied.

It is easy to see that there are sequences $\left\{a_{n}\right\}$ which satisfy the hypothesis of Corollary 2 but for which $\liminf _{n \rightarrow \infty} \frac{a_{n+1}}{a_{n}}=0$.

We have included an expanded list of references. 


\section{REFERENCES}

1. D. Borwein and S. Z. Ditor, Translates of sequences in sets of positive measure, Canad. Math. Bull. 21 4, (1978), 497-498. MR 80i:28018

2. S. J. Eigen, Putting convergent sequences into measurable sets, Studia Sci. Math. Hung. 20 (1985), 411-412. MR 88f:28003

3. P. Erdős, Problems, Math. Balkanica 4 (1974), 203-204. MR 55:2715

4. - My Scottish Book 'problems', The Scottish Book (R. D. Mauldin, Ed.), Birkhäuser, Boston, (1981), 35-43. MR 84m:00015

5. __ Set theoretic, measure theoretic, combinatorial, and number theoretic problems concerning point sets in Euclidean space, Real Anal. Exch. 4 (1978/'79), 113-138.

6. Some measure theoretic problems of a combinatoric and geometric nature, Measure Theory Conference at Oberwolfach, (1983). MR 85e:28008

7. K. J. Falconer, On a problem of Erdös on sequences and measurable sets, Proc. Amer. Math. Soc. 90 (1984), 77-78. MR 85e:28008

8. P. Komjáth, Large sets not containing images of a given sequence, Canad. Math. Bull. 26 (1983), 41-43. MR 85d:28003

9. H. I. Miller, Some results connected with a problem of Erdös, II, Proc. Amer. Math. Soc. 75 (1979), 265-268. MR 82c:28003

10. J. Arias de Reyna, Some results connected to a problem of Erdös, III, Proc. Amer. Math. Soc. 89 (1983), 291-292. MR 85d:28001

Department of Mathematics, St. Olaf College, Northfield, Minnesota 55057

E-mail address: humke@stolaf.edu

Department of Analysis, Eötvös Loránd University, Múzeum krt. 6-8, Budapest H1088, Hungary

E-mail address: laczk@cs.elte.hu 